\title{
(Un)happy doctors? - zwischen Arzt-Sein und Managen
}

\author{
P. Berchtold, C. Schmitz
}

Wenn wir «managen» hören, denken viele erst mal an Betriebswirtschaft, Geld und aktuell vor allem ans Sparen. Und gerade das löst Sorgen aus, beispielsweise um die ärztliche Autonomie, um die Behandlungsqualität oder die Aus- und Weiterbildung. Diese Engführung von Management mit Ökonomie ist verständlich, wurde Management doch wesentlich in Wirtschaftsorganisationen entwickelt. Mittlerweile haben die Managementwissenschaften aber diese enge ökonomische Ausrichtung überwunden und orientieren sich heute interdisziplinär, u.a. unter Einbeziehung von Soziologie, Informatik, Kommunikationswissenschaft, Philosophie, Psychologie, Ethik usw. Management ist heute eine gesellschaftliche Leitdisziplin und hat einen entsprechend hohen Stellenwert. Überall wird «gemanaged» und geführt. Kaderpersonen von Unternehmen investieren einen Grossteil ihrer Arbeitszeit in Management und Führung und auch andere gesellschaftliche Bereiche wie NonProfit-Organisationen oder Verwaltung können sich der Sogwirkung des Managements nicht entziehen.

Und in den Gesundheits- und Spitalinstitutionen? Eine neue in der Schweizerischen Ärztezeitung veröffentlichte Studie befragte Chefärzte und Chefärztinnen in der ganzen Schweiz, wieviel ihrer Arbeitszeit sie für Führung und Management aufwenden. Das Resultat: durchschnittlich unter 10 Stunden pro Woche oder etwa 10\% der Arbeitszeit! [1] Jeder CEO irgendeines Unternehmens würde sich dazu verwundert fragen, wie so etwas sein kann und wo denn dann eigentlich Management und Führung stattfinden oder welche Beziehung (bzw. Abgrenzung) zwischen Management und dem professionellen Selbstverständnis der Experten besteht?

Korrespondenz: PD Dr. med. Peter Berchtold Dr. Christof Schmitz College-M Freiburgstrasse 41 CH-3010 Bern nehmende Spezialisierung sind nur mit einem ebenfalls grösseren Steuerungs- oder Koordinationsaufwand $\mathrm{zu}$ meistern. Verwaltung alleine reicht nicht mehr aus. Management kommt ins Spiel - und ist nicht mehr wegzudenken. Wie aber vertragen sich Management und Medizin? Wie wir aus der Organisations- und Führungsforschung wissen, wollen sich professionelle Organisationen nur in begrenztem Ausmass Führung zumuten lassen. Was für Industrie oder Banken gilt, funktioniert nicht gleichermassen für Organisationen wie Behörden, Armeen, Opernhäuser, Redaktionen, Schulen, Universitäten und nicht zuletzt Krankenhäuser. Diese verzichten gerne auf Führung und setzen stattdessen auf die Inszenierung und Verwaltung des professionellen Selbstverständnisses. Warum ist das so? Professionen reagieren ungnädig, wenn ihr Selbstverständnis und damit auch ihre Autorität hinterfragt wird. Führung droht jedoch - früher oder später -, eben das zu tun. Denn Führung sucht zwangsläufig nach Ungewissheiten, die zum Nährboden der nächsten Innovationen oder Optimierungen werden können.

Die klassische strategische Managementfrage «What business are we in?» kann damit in professionellen Kontexten nur mit Schwierigkeiten gestellt werden. Denn diese Frage bezieht sich auf die Definitions-, Gestaltungs- und Innovationsmöglichkeit, die in ihrer Beantwortung wettbewerbsrelevante Unterschiede hervorbringt. Wird jedoch die besagte Ungewissheit in professionellen Organisationen wie Spitälern negiert, ergibt sich «ein Verzicht sowohl auf das Selbstverständnis als Organisation als auch auf den Bedarf an Führung» [2]. Das ging so lange gut, wie das professionelle Selbstverständnis nicht von aussen, beispielsweise via Kosten, hinterfragt wurde. Heute wissen wir, dass auch im Gesundheitswesen vielfältige soziale und ökonomische Gründe zwingen, Führung und Management zu akzeptieren. Dieser Prozess läuft unumkehrbar, wenn auch nicht reibungslos. «The unhappy doctor» kann die Folge sein, wie in England seit einiger Zeit Arzt-Sein unter eben diesen veränderten Rahmenbedingungen diskutiert wird [3]. 


\section{... une danse macabre?}

Der Bedeutungszuwachs von Management und Führung verläuft offenkundig nicht konfliktlos. Denn aus der Organisationsforschung wissen wir, dass Expertenorganisationen durch eine ihnen eigene Dynamik gekennzeichnet sind, die aus dem Ringen um Einfluss durch die sie konstituierenden Subgruppen entsteht [4]. In Gesundheitsinstitutionen sind diese Subgruppen typischerweise:

- Experten (z. B. Ärzte, Pflege);

- Leader (z. B. Klinik oder Spitalleitungen);

- Manager (z. B. Controller);

- Supportpersonal.

In professionellen Organisationen spielen natürlich die Experten die zentrale Rolle. Denn sie sind es, die das Kerngeschäft betreiben und die mit ihrem Selbstverständnis die Organisation prägen. Indem sich Führung (via Leader) stärker behauptet, beginnen sich die Kräfteverhältnisse in der Organisation zu verschieben. Die grosse Frage tut sich auf, ob dieses Ringen zur Konfrontation gerät oder ob es gelingt, die Experten in Managementrollen $\mathrm{zu}$ involvieren und damit Experten- mit Managementwissen zu verschmelzen. Gelingt dies nicht, tun sich für alle hinderliche Konfrontationen auf. Medizinische Fachpersonen erleben dann Management oftmals als unfachliches und unangemessenes Eingreifen in ihre professionellen Sphären. Sie fühlen sich und ihre Anliegen nicht ausreichend berücksichtigt und sehen sich gegebenenfalls in ihrer Autonomie bedroht. Verständlich, dass sie darauf reagieren. Die daraus resultierenden Konflikte münden leicht in eine Polarisierung von Medizin und Management. Da stehen sich dann Patientenwohl und Sparen gegenüber, oder Heilen und Verdienen.

Diese Symptomatik findet sich, wie unsere Forschung in der Schweiz, Deutschland wie England zeigt, überall in ähnlicher Art und Weise [5]. Das ist durchaus bemerkenswert, denn bei allen unterschiedlichen Gesamtkonstruktionen der Gesundheitssysteme ergeben sich hinsichtlich der Führungsdynamik und der Spannung zwischen Medizin und Management kaum Unterschiede. Dieser Befund findet Bestätigung in Studien, die zeigen, dass die berufsgruppenspezifischen, professionellen Orientierungen wichtiger als national-kulturelle oder gesundheitssystembezogene Faktoren sind [6].

\section{... oder doch möglich?}

Dass das Gesundheitssystem im Wandel ist, wissen wir. Der Wandel bezieht sich dabei nicht nur auf die Kostenseite, sondern reicht viel weiter, wie z.B. der Ruf nach evidenzbasierter Medizin, die Forderung nach ausgewiesenen Grenznutzen neuer medizinischer Massnahmen oder die Diskussion um den «mündigen Patienten» erahnen lassen. Es ist nicht einfach Management, das auf «abwegige» Gedanken kommt, sondern es ist die Gesellschaft, die hinsichtlich Ethik, Kosten usw. die professionelle Autonomie der Experten hinterfragt. Management spielt eine wichtige Rolle in der Aushandlung und Ausgestaltung dieses Wandels. Die Beziehungsgestaltung Medizin Management wird zur alltäglichen Herausforderung für Führungspersonen in Spitälern. In dieser Situation sind empfehlenswerte Strategien:

- Ein sorgfältiger Einbezug von Ärzten ins Management, da andernfalls für alle hinderliche Polarisierungen drohen.

- Das intensive Verfolgen und Schulen integrativer Führungsansätze, die sich der Bedeutung der Prozesshaftigkeit von Management stellen und Praktiken (er)finden, die den prekären Einflussverhältnissen Rechnung tragen.

- Ein Management mit hoher kultureller Sensibilität, um die nötigen Brücken bauen zu können und zu vermeiden, in die verschiedentlich bereitstehenden Fallen (der Ungeduld, der Abwertung und der Kategorisierungen) zu laufen.

\section{Literatur}

1 Angehrn A, Eichenberger T, Wyss F. Anstellungsbedingungen der Kaderärztinnen und Kaderärzte an öffentlichen und öffentlich subventionierten Spitälern. Schweiz Ärztezeitung 2004;85(51/52): 2754-7.

2 Baecker D. Organisation und Management. Frankfurt: Suhrkamp; 2002.

3 Edwards N, Kornacki MJ, Silversin J. Unhappy doctors: what are the causes and what can be done? Br Med J 2002;324:835-8.

4 Sveiby K. The New Organizational Wealth. San Francisco: Wiley; 1997.

5 Forschungsprojekt «Managing Professionals / Professionals managing», finanziert durch die WHI (Winterthur Health Initiative).

6 Degeling P, Maxwell S, Kennedy J, Coyle B. Medicine, management, and modernisation: a «danse macabre»? Br Med J 2003;326:649-52. 